\title{
Phytosociological Studies on Apis cerana F. and Apis mellifera L. in Shiwalik Hills
}

\author{
Avinash Kaur ${ }^{1}$, V. K. Mattu ${ }^{2}$ \\ Sociobiology and Behavioral Ecology Research Lab, Department of Biosciences, Himachal Pradesh University, Shimla (H.P)-171005, India
}

\begin{abstract}
In India beekeeping is still in infancy, offering vast scope for expansion as a prime agri-horticulture and forest-based rural industry. In order to make beekeeping a successful enterprise, first and foremost task in India is to educate beekeepers about the bee pasturage availability in different agroclimatic zones. This provides valuable information on availability and abundance of pollen and nectar sources, for honeybees during different seasons and helps ultimately in standardizing the seasonal management practices for honey bee colony. Unfortunately, our knowledge on this aspect in the country is scanty and unorganized. Therefore, phytosociological studies were conducted in shiwalik hills. Observations on the bee flora of shiwalik hills were made in the blooming period.These investigations revealed that predominant honey plants were: Brassica sp. Raphanus sp. Murraya sp Eucalyptus sp. etc. whereas, calendula sp., Mangifera sp, Eruca sp., Trifolium sp., Woodfordia sp.. Psidium sp., Grewia sp., Phoenix sp., Hibiscus sp., Syzygium sp., Melia sp., Grewia sp., Acacia sp., Adhatoda sp., Citrus sp., Azadirachta sp. etc. were secondary pollen types. The important minor and minor pollen components represented in these honey samples were: Acacia sp., Adhatoda sp., Ageratum sp., Allium sp., Bauhinia sp., Berberis sp., Bidens sp., Callistemon sp., Carissa sp., Cassia sp., Chenopodium sp., Dalbergia sp., Dianthus sp., Gossypium sp., Grevillea sp., Grewia sp., Helianthus sp., Lagerstroemia sp., Lilium sp., Medicago sp., Murraya sp., Rubus sp., Salvia sp., Sapindus sp., Syzygium sp., Zea sp., Zizyphus sp., Bauhinia sp., Cestrum sp., Citrus sp., Litchi sp., Raphanus sp. Bombax sp., Embilica sp., Pisum sp., Rosa sp., Woodfordia sp. Aegle sp., Albizzia sp., Berberis sp.,Calendula sp., Cedrella sp., Dianthus sp., Leucaena sp., Ocimum sp., Pisum sp., Polygonum sp., Punica sp., Ricinus sp., Robinia sp., Taraxacum sp., Sonchus sp., Syzygium sp., Vitis sp., Bombax sp., Litchi sp and members of families Boraginaceae, Convolvulaceae, Euphorbiaceae, Fabaceae, Meliaceae, Asteraceae, Cucurbitaceae Myrtaceae Acanthaceae, Apiaceae, Apocynaceae, Boraginaceae, Brassicaceae, Cannabinaceae, Lamiaceae, Malvaceae, Papaveraceae, Poaceae, Rosaceae ,Sapindaceae and Rutaceae. Most of the honey samples had pollen of entomophilous plants with a small percentage of anemophilous types. These investigations also revealed both uniflorality and multiflorality in different honey samples. On the basis of present bee botanical investigations, a floral calendar of honey plant resources of Shiwalik hills indicating their taxonomic status, geographic location, honey potentiality, periods of flowering and economic uses has been prepared. Present studies thus suggest that this region offers very rich potential for the development of beekeeping due to the multiplicity of bee flora available throughout the year. Such investigations can be helpful in setting up new apiaries, in migratory beekeeping practices and application of modern bee management technology.
\end{abstract}

Keywords: Beekeeping, Honey, Phytosociology, Pollen, Nectar, Bee flora

\section{Introduction}

Most of the flowering plants are pollinated by insects and many insects are specific to certain species of plants. Honey bees, on the other hand have no such restriction of pollen and nectar (McGregor, 1976). There are at least four hundred species of plants which are either major or minor sources of pollen and nectar to Indian hive bee, Apis cerana F. (Deodikar and Suryanarayana, 1972; Crane and Walker, 1984). It is obvious that all the plant species are not available in any one locality and a given plant species may also show variations in its utility to bees in different localities or during different years. Information on the utility of each of these plants species, its distribution, abundance and preferences for any climate or soil etc. is of great importance to beekeepers (Suryanarayana, 1978; Mattu, 1982).

Shiwalik hills are the principle temperate and tropical fruit growing region of the country. Every year more and more land is coming under cultivation in this region. With the knowledge of major bee plants of any given place and at any given time, the migratory bee keeping can be scientifically founded.
Phytosociological studies have been carried out in different parts of India by various investigated and fairly good lists of bee plants. However, very little work has been done to identify and evaluate the important honey plants of shiwalik hills, which has great potential for beekeeping. The present studies are a step in the direction of knowing and working out the floral resources of shiwalik hills.

\section{Materials \& Methods}

Observations on the bee flora of Shiwalik hills were made during October, 2008 to September, 2009. Observations on blooming of different plant sources were recorded at weekly intervals. The period of activity of honey bees on a blooming crop was taken as the flowering period of that particular plant species.

The plants at flowering stage near an apiary were visited. The plants which yielded good pollen packs were listed as pollen sources. The abundance of nectar secretion from a large number of plants in a particular situation was taken as the honey flow period, whereas the scarcity of food for the bees was recorded as a dearth period. Definite durations of food scarcity were worked out from the list of bee attracting plants recorded throughout the year. 


\section{International Journal of Science and Research (IJSR) \\ ISSN (Online): 2319-7064}

Index Copernicus Value (2013): 6.14 | Impact Factor (2015): 6.391

\section{Source of bee forage during different months of the year in shiwalik hills}

\begin{tabular}{|c|c|c|c|c|}
\hline Name of the Plant & & Family & $\begin{array}{l}\text { Source of } N \\
\text { or P or both }\end{array}$ & Economic importance \\
\hline \multicolumn{5}{|l|}{ January } \\
\hline Dahlia pinnata & Dahlia & Asteraceae & $\mathrm{N}^{2} \mathrm{p}^{2}$ & Ornamental \\
\hline Brassica campestris & Mustard & Brassicaceae & $\mathrm{N}^{1} \mathrm{P}^{1}$ & Oilseed crop \\
\hline B. campestris var. sarson & Sarson & Brassicaceae & $\mathrm{N}^{1} \mathrm{P}^{1}$ & Oilseed crop, Vegetable \\
\hline Brassica napus var. glauca & Rape & Brassicaceae & $\mathrm{N}^{1} \mathrm{P}^{1}$ & Oilseed crop, Vegetable \\
\hline Brassica napus var. toria & Toria & Brassicaceae & $\mathrm{N}^{1} \mathrm{P}^{1}$ & Oilseed crop, Vegetable \\
\hline Eruca sativa & Rocket salad & Brassicaceae & $\mathrm{N}^{1} \mathrm{P}^{1}$ & Oilseed, Fodder, \\
\hline Malvaviscus arboreus & Malvaviscus & Malvaceae & $\mathrm{N}^{2} \mathrm{p}^{2}$ & Ornamental \\
\hline Moringa oleifera & Drumstick tree & Moringaceae & $\mathrm{N}^{1} \mathrm{P}^{1}$ & $\begin{array}{l}\text { Perfumes and Lubricants, Fertilizer, Gum, } \\
\text { Fodder,Vegetable, Antibacterial properties }\end{array}$ \\
\hline \multicolumn{5}{|l|}{ February } \\
\hline Centaurea cyanus & Cornflower & Asteraceae & $\mathrm{N}^{1} \mathrm{P}^{1}$ & Ornamental \\
\hline Helichrysum arenarium & Paperflower & Asteraceae & $\mathrm{N}^{2} \mathrm{p}^{3}$ & Ornamental \\
\hline Bombax ceiba & Silk Cotton tree & Bombacaceae & $\mathrm{N}^{1} \mathrm{P}^{1}$ & Fodder, Tree, Fibre, Timber \\
\hline Brassica campestris & Mustard & Brassicaceae & $\mathrm{N}^{1} \mathrm{P}^{1}$ & Oilseed crop, Vegetable \\
\hline B. campestris var. sarson & Sarson & Brassicaceae & $\mathrm{N}^{1} \mathrm{P}^{1}$ & Oilseed crop, Vegetable \\
\hline Brassica juncea & Indian mustard & Brassicaceae & $\mathrm{N}^{1} \mathrm{P}^{1}$ & Oilseed crop \\
\hline Brassica napus var. glauca & Rape & Brassicaceae & $\mathrm{N}^{1} \mathrm{P}^{1}$ & Oilseed crop, Vegetable \\
\hline Brassica napus var. toria & Toria & Brassicaceae & $\mathrm{N}^{1} \mathrm{P}^{1}$ & Oilseed crop, Vegetable \\
\hline Eruca sativa & Rocket salad & Brassicaceae & $\mathrm{N}^{1} \mathrm{P}^{1}$ & Oilseed, Fodder \\
\hline Raphanus sativus & Radish & Brassicaceae & $\mathrm{N}^{1} \mathrm{P}^{1}$ & Vegetable \\
\hline Cucurbita maxima & Great Pumpkin & Cucurbitaceae & $\mathrm{N}^{1} \mathrm{P}^{1}$ & Vegetable \\
\hline Bauhinia variegata & Kachnar & Fabaceae & $\mathrm{N}^{2} \mathrm{P}^{3}$ & Fodder, Fuel, Ornamental, Vegetable \\
\hline Malvaviscus arboreus & Malvaviscus & Malvaceae & $\mathrm{N}^{2} \mathrm{p}^{2}$ & Ornamental \\
\hline Moringa oleifera & Drum stick tree & Moringaceae & $\mathrm{N}^{1} \mathrm{P}^{1}$ & $\begin{array}{l}\text { Perfumes and Lubricants, Fertilizer, Gum, } \\
\text { Fodder,Vegetable, Antibacterial properties }\end{array}$ \\
\hline Salix babylonica & Weaping willow & Salicaceae & $\mathrm{N}^{1} \mathrm{P}^{1}$ & Timber \\
\hline Litchi chinensis & Litchi & Sapindaceae & $\mathrm{N}^{1} \mathrm{P}^{1}$ & Fruit tree \\
\hline March & & +2 & $-\quad+2$ & \\
\hline Mangifera indica & Mango & Anacardiaceae & $\mathrm{N}^{3} \mathrm{P}^{3}$ & Fruit tree, Fuel, Timber \\
\hline Daucus carota & Carrot & Apiaceae & $\mathrm{N}^{2} \mathrm{P}^{3}$ & Vegetable \\
\hline Centaurea cyanus & Cornflower & Asteraceae & $\mathrm{N}^{1} \mathrm{P}^{1}$ & Ornamental \\
\hline Helichrysum arenarium & Paper flower & Asteraceae & $\mathrm{N}^{2} \mathrm{P}^{3}$ & Ornamental \\
\hline Taraxacum officinale & Dandelion & Asteraceae & $\mathrm{N}^{1} \mathrm{P}^{1}$ & Juicy Weed \\
\hline Bombax ceiba & Silk cotton tree & Bombacaceae & $\mathrm{N}^{1} \mathrm{P}^{1}$ & Fodder, Tree, Fibre, Timber \\
\hline Brassica campestris & Mustard & Brassicaceae & $\mathrm{N}^{1} \mathrm{P}^{1}$ & Oilseed crop \\
\hline B.Campestris var. sarson & Sarson & Brassicaceae & $\mathrm{N}^{1} \mathrm{P}^{1}$ & Oilseed crop, Vegetable \\
\hline Brassica juncea & Indian mustard & Brassicaceae & $\mathrm{N}^{1} \mathrm{P}^{1}$ & Oilseed crop, \\
\hline Brassica napus var. glauca & Rape & Brassicaceae & $\mathrm{N}^{1} \mathrm{P}^{1}$ & Oilseed crop, Vegetable \\
\hline Brassica napus var. toria & Toria & Brassicaceae & $\mathrm{N}^{1} \mathrm{P}^{1}$ & Oilseed crop, Vegetable \\
\hline Brassica oleracea capitata & Cabbage & Brassicaceae & $\mathrm{N}^{2} \mathrm{P}^{2}$ & Vegetable \\
\hline Brassica oleracia botrytis & Cauliflower & Brassicaceae & $\mathrm{N}^{2} \mathrm{P}^{2}$ & Vegetable \\
\hline Brassica rapa & Turnip & Brassicaceae & $\mathrm{N}^{2} \mathrm{P}^{2}$ & Vegetable \\
\hline Eruca sativa & Rocket salad & Brassicaceae & $\mathrm{N}^{1} \mathrm{P}^{1}$ & Oilseed, Fodder \\
\hline Raphanus sativus & Radish & Brassicaceae & $\mathrm{N}^{1} \mathrm{P}^{1}$ & Vegetable \\
\hline Chenopodium album & White goose foot & Chenopodiaceae & $\mathrm{N}^{3} \mathrm{P}^{3}$ & Fodder \\
\hline Cucurbita maxima & Great Pumpkin & Cucurbitaceae & $\mathrm{N}^{1} \mathrm{P}^{1}$ & Vegetable \\
\hline Emblica officinalis & Indian goose berry & Euphorbiaceae & $\mathrm{N}^{2} \mathrm{P}^{2}$ & Medicinal \\
\hline Bauhinia vahlii & Camel'sfoot & Fabaceae & $\mathrm{N}^{2} \mathrm{P}^{3}$ & Fodder, Fuel, Vegetable \\
\hline Bauhinia variegata & Kachnar` & Fabaceae & $\mathrm{N}^{2} \mathrm{P}^{3}$ & Fodder, Fuel, Ornamental,Vegetable \\
\hline Dalbergia sissoo & Sissoo & Fabaceae & $\mathrm{N}^{1} \mathrm{P}^{1}$ & Timber, Fuel, Fodder, Shade \\
\hline Melilotus spp. & Mellilot & Fabaceae & $\mathrm{N}^{1} \mathrm{P}^{2}$ & Fodder \\
\hline Pisum sativum & Garden Pea & Fabaceae & $\mathrm{N}^{3} \mathrm{P}^{3}$ & Vegetable \\
\hline Tamarindus indica & Tamarind & Fabaceae & $\mathrm{N}^{3} \mathrm{P}^{3}$ & Avenue tree, Condiment, Fruit, Oil \\
\hline Woodfordia fruticosa & Dhawi & Lytheraceae & $\mathrm{N}^{1} \mathrm{P}^{1}$ & Dye, Medicinal, Fodder, Fuel \\
\hline Hibiscus rosa-sinensis & Chinese rose & Malvaceae & $\mathrm{N}^{2} \mathrm{P}^{2}$ & Ornamental \\
\hline Malvaviscus arboreus & Malvaviscus & Malvaceae & $\mathrm{N}^{2} \mathrm{P}^{2}$ & Ornamental \\
\hline Cedrella toona & Cedrella & Meliaceae & $\mathrm{N}^{1} \mathrm{P}^{2}$ & Furniture \\
\hline Morus alba & Mulberry & Moraceae & $\mathrm{P}^{2}$ & Silkworm rearing, Fruits edible \\
\hline Moringa oleifera & Drumstick tree & Moringaceae & $\mathrm{N}^{1} \mathrm{P}^{1}$ & $\begin{array}{l}\text { Tree, Perfumes and Lubricants, Fertilizer, Gum, } \\
\text { Fodder,Vegetable, Antibacterial properties }\end{array}$ \\
\hline Musa sapientum & Banana & Musaceae & $\mathrm{N}^{1} \mathrm{P}^{3}$ & Fruit \\
\hline Callistemon citrinus & Bottle brush & Myrtaceae & $\mathrm{N}^{1} \mathrm{P}^{1}$ & Ornamental \\
\hline Eschscholzia californica & Californian poppy & Papaveraceae & $\mathrm{N}^{3} \mathrm{p}^{1}$ & Ornamental \\
\hline
\end{tabular}


International Journal of Science and Research (IJSR)

ISSN (Online): 2319-7064

Index Copernicus Value (2013): 6.14 | Impact Factor (2015): 6.391

\begin{tabular}{|c|c|c|c|c|}
\hline Papaver rhoeas & Poppy & Papaveraceae & $\mathrm{N}^{3} \mathrm{p}^{3}$ & Ornamental \\
\hline Plantago spp. & Plantago & Plantaginaceae & $\mathrm{N}^{2} \mathrm{p}^{1}$ & - \\
\hline Delphinium roylei & Larkspur & Ranunculaceae & $\mathrm{N}^{3} \mathrm{p}^{3}$ & Ornamental \\
\hline Rosa macrophylla & Rose & Rosaceae & $\mathrm{N}^{3} \mathrm{p}^{1}$ & Ornamental \\
\hline Citrus aurantifolia & Lemon & Rutaceae & $\mathrm{N}^{1} \mathrm{P}^{1}$ & Fruit Tree \\
\hline Citrus grandis & Pumelo & Rutaceae & $\mathrm{N}^{1} \mathrm{P}^{1}$ & Fruit Tree \\
\hline Citrus limetta & Sweet lemon & Rutaceae & $\mathrm{N}^{1} \mathrm{P}^{1}$ & Fruit Tree \\
\hline Citrus medica & Citron & Rutaceae & $\mathrm{N}^{1} \mathrm{P}^{1}$ & Fruit Tree \\
\hline Citrus reticulata & Mandarin orange & Rutaceae & $\mathrm{N}^{1} \mathrm{P}^{1}$ & Fruit Tree \\
\hline Citrus sinensis & Sweet orange & Rutaceae & $\mathrm{N}^{1} \mathrm{P}^{1}$ & Fruit Tree \\
\hline Murraya koenigii & Curry leaf plant & Rutaceae & $\mathrm{N}^{2} \mathrm{P}^{2}$ & Medicinal, Ornamental \\
\hline Salix babylonica & Weeping willow & Salicaceae & $\mathrm{N}^{1} \mathrm{P}^{1}$ & Timber \\
\hline Litchi chinensis & Litchi & Salicaceae & $\mathrm{N}^{1} \mathrm{P}^{1}$ & Fruit Tree \\
\hline Lycopersicum esculentum & Tomato & Solanaceae & $\mathrm{N}^{3} \mathrm{P}^{3}$ & Vegetable \\
\hline \multicolumn{5}{|l|}{ April } \\
\hline Adhatoda vasica & Basuti & Acanthaceae & $\mathrm{N}^{2} \mathrm{P}^{2}$ & - \\
\hline Mangifera indica & Mango & Anacardiaceae & $\mathrm{N}^{3} \mathrm{P}^{3}$ & Fruit tree, Fuel, Timber \\
\hline Daucus carota' & Carrot & Apiaceae & $\mathrm{N}^{2} \mathrm{P}^{3}$ & Vegetable \\
\hline Carissa caranda & Karandas & Apocynaceae & $\mathrm{N}^{2} \mathrm{P}^{2}$ & Preservation \\
\hline Asclepias curassavica & Milk weed & Asclepiadaceae & $\mathrm{N}^{2} \mathrm{P}^{2}$ & Fibre \\
\hline Centaurea cyanus & Cornflower & Asteraceae & $\mathrm{N}^{1} \mathrm{P}^{1}$ & Ornamental \\
\hline Helichrysum arenarium & Paper flower & Asteraceae & $\mathrm{N}^{2} \mathrm{P}^{3}$ & Ornamental \\
\hline Taraxacum officinale & Dandelion & Asteraceae & $\mathrm{N}^{1} \mathrm{P}^{1}$ & Juicy Weed \\
\hline Tussilago farfara & Coltsfoot & Asteraceae & $\mathrm{N}^{3} \mathrm{P}^{3}$ & Medicinal \\
\hline Berberis aristata & Berberry & Berberidaceae & $\mathrm{N}^{2} \mathrm{P}^{2}$ & Fruit, Dye, Medicinal \\
\hline Berberis lycium & Raisin berberry & Berberidaceae & $\mathrm{N}^{2} \mathrm{P}^{2}$ & Fruit, Medicinal \\
\hline Ehretia acuminata & Ivory wood & Boraginaceae & $\mathrm{N}^{1} \mathrm{P}^{2}$ & Avenue tree, Fruit, Timber, Fodder \\
\hline Brassica campestris & Mustard & Brassicaceae & $\mathrm{N}^{1} \mathrm{P}^{1}$ & Oilseed crop \\
\hline Brassica rapa & Turnip & Brassicaceae & $\mathrm{N}^{2} \mathrm{P}^{2}$ & Vegetable \\
\hline Opuntia spp. & Prickly pear & Cactaceae & $\mathrm{N}^{2} \mathrm{P}^{2}$ & - \\
\hline Dianthus caryophyllus & Carnation & Caprifoliaceae & $\mathrm{N}^{3} \mathrm{P}^{3}$ & Ornamental \\
\hline Chenopodium album & White goose foot & Chenopodiaceae & $\mathrm{N}^{3} \mathrm{P}^{3}$ & Fodder \\
\hline Terminalia spp. & Myrobalan & Combretaceae & $\mathrm{N}^{1} \mathrm{P}^{1}$ & $\begin{array}{c}\text { Timber, Fruit, Medicinal, Fuel, Bark for tanning, } \\
\text { Dyeing }\end{array}$ \\
\hline Convolvulus arvensis & Convolvulus & Convolvulaceae & $\mathrm{N}^{3} \mathrm{P}^{3}$ & 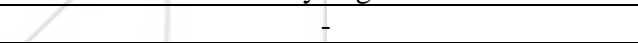 \\
\hline Benincasa spp. & Petha & Cucurbitaceae & $\mathrm{N}^{2} \mathrm{P}^{2}$ & Vegetable \\
\hline Cucurbita pepo. & Pumpkin & Cucurbitaceae & $\mathrm{N}^{1} \mathrm{P}^{1}$ & Vegetable \\
\hline Cucurbita maxima & Great pumpkin & Cucurbitaceae & $\mathrm{N}^{1} \mathrm{P}^{1}$ & Vegetable \\
\hline Emblica officinalis & Indian gooseberry & Euphorbiaceae & $\mathrm{N}^{2} \mathrm{P}^{2}$ & Medicinal \\
\hline Euphorbia royleana & Euphorbia & Euphorbiaceae & $\mathrm{N}^{3} \mathrm{P}^{3}$ & Juicy \\
\hline Acacia catechu & Acacia & Fabaceae & $\mathrm{N}^{1} \mathrm{P}^{1}$ & Timber, Fodder, Fuel-wood \\
\hline Albizzia lebbek & Siris & Fabaceae & $\mathrm{N}^{3} \mathrm{P}^{3}$ & Fodder, Timber, Fuel \\
\hline Bauhinia vahlii & Camel's foot & Fabaceae & $\mathrm{N}^{2} \mathrm{P}^{3}$ & Fodder, Fuel, Vegetable \\
\hline Bauhinia variegata & Kachnar & Fabaceae & $\mathrm{N}^{2} \mathrm{P}^{3}$ & Fodder, Fuel, Ornamental, Vegetable \\
\hline Cassia fistula & Indian laburnum & Fabaceae & $\mathrm{N}^{2} \mathrm{P}^{2}$ & Avenue tree \\
\hline Tamarindus indica & Tamarind & Fabaceae & $\mathrm{N}^{3} \mathrm{P}^{3}$ & Avenue tree, Condiment, Fruit, Fodder, Oil \\
\hline Trifolium alexandrinum & Egyptian clover & Fabaceae & $\mathrm{N}^{1} \mathrm{P}^{1}$ & Fodder \\
\hline Trifolium pretense & Red clover & Fabaceae & $\mathrm{N}^{1} \mathrm{P}^{2}$ & Fodder \\
\hline Vicia spp. & Field beans & Fabaceae & $\mathrm{N}^{3} \mathrm{P}^{3}$ & $\begin{array}{l}\text { Edible, Fodder } \\
\end{array}$ \\
\hline Hypericum spp. & Hypericum & Hypericaceae & $\mathrm{P}^{1}$ & $\begin{array}{l}\text { Twigs used as toothbrush, seed as condiment for } \\
\text { flavouring curries }\end{array}$ \\
\hline Woodfordia fruticosa & Dhawi & Lytheraceae & $\mathrm{N}^{1} \mathrm{P}^{1}$ & Dye, Medicinal, Fodder, Fuel \\
\hline Hibiscus rosa-sinensis & Chinese rose & Malvaceae & $\mathrm{N}^{2} \mathrm{P}^{2}$ & Ornamental \\
\hline Malvaviscus arboreus & Malvaviscus & Malvaceae & $\mathrm{N}^{2} \mathrm{P}^{2}$ & Ornamental \\
\hline Cedrella toona & Cedrella & Meliaceae & $\mathrm{N}^{1} \mathrm{P}^{2}$ & Woody, Furniture \\
\hline Morus alba & Mulberry & Moraceae & $\mathrm{P}^{2}$ & Silkworm rearing, Fruits edible \\
\hline Musa sapientum & Banana & Musaceae & $\mathrm{N}^{2} \mathrm{P}^{3}$ & Fruit \\
\hline Callistemon citrinus & Bottle brush & Myrtaceae & $\mathrm{N}^{1} \mathrm{P}^{1}$ & Ornamental \\
\hline Syzygium cumini & Jambolan & Myrtaceae & $\mathrm{N}^{1} \mathrm{P}^{1}$ & Avenue tree, Fruit \\
\hline Grevillea robusta & Silky oak & Proteaceae & $\mathrm{N}^{1} \mathrm{P}^{1}$ & Avenue tree, Ornamental, Timber \\
\hline Punica granatum & Pomegranate & Punicaceae & $\mathrm{N}^{2} \mathrm{P}^{1}$ & Fruit \\
\hline Anemone spp. & Wood anemone & Ranunculaceae & $\mathrm{P}^{3}$ & Ornamental \\
\hline Clematis spp. & Clematis & Ranunculaceae & $\mathrm{N}^{2} \mathrm{P}^{2}$ & Medicinal \\
\hline Delphinium roylei & Larkspur & Ranunculaceae & $\mathrm{N}^{3} \mathrm{P}^{3}$ & Ornamental \\
\hline Rosa macrophylla & Rose & Rosaceae & $\mathrm{N}^{3} \mathrm{P}^{1}$ & Ornamental \\
\hline Rosa moschata & Wild rose & Rosaceae & $\mathrm{N}^{3} \mathrm{P}^{1}$ & Ornamental \\
\hline Rubus spp. & Berries & Rosaceae & $\mathrm{N}^{2} \mathrm{P}^{2}$ & Fruit \\
\hline
\end{tabular}




\section{International Journal of Science and Research (IJSR) ISSN (Online): 2319-7064}

Index Copernicus Value (2013): 6.14 | Impact Factor (2015): 6.391

\begin{tabular}{|c|c|c|c|c|}
\hline Citrus aurantifolia & Lemon & Rutaceae & $\mathrm{N}^{1} \mathrm{P}^{1}$ & Fruit tree \\
\hline Citrus grandis & Pumelo & Rutaceae & $\mathrm{N}^{1} \mathrm{P}^{1}$ & Fruit tree \\
\hline Citrus limetta & Sweet lemon & Rutaceae & $\mathrm{N}^{1} \mathrm{P}^{1}$ & Fruit tree \\
\hline Citrus medica & Citron & Rutaceae & $\mathrm{N}^{1} \mathrm{P}^{1}$ & Fruit tree \\
\hline Citrus reticulata & Mandarin orange & Rutaceae & $\mathrm{N}^{1} \mathrm{P}^{1}$ & Fruit tree \\
\hline Citrus sinensis & Sweet orange & Rutaceae & $\mathrm{N}^{1} \mathrm{P}^{1}$ & Fruit tree \\
\hline Murraya koenigii & Curry leaf plant & Rutaceae & $\mathrm{N}^{2} \mathrm{P}^{2}$ & Medicinal, Ornamental \\
\hline Lycopersicum esculentum & Tomato & Solanaceae & $\mathrm{N}^{3} \mathrm{P}^{3}$ & Vegetable \\
\hline \multicolumn{5}{|l|}{ May } \\
\hline Adhatoda vasica & Basuti & Acanthaceae & $\mathrm{N}^{2} \mathrm{P}^{2}$ & - \\
\hline Allium cepa & Onion & Amaryllidaceae & $\mathrm{N}^{3} \mathrm{P}^{3}$ & Vegetable \\
\hline Allium sativum & Garlic & Amaryllidaceae & $\mathrm{N}^{3} \mathrm{P}^{3}$ & Vegetable \\
\hline Coriandrum sativum & Coriander & Apiaceae & $\mathrm{N}^{3} \mathrm{P}^{3}$ & Condiment \\
\hline Daucus carota & Carrot & Apiaceae & $\mathrm{N}^{2} \mathrm{P}^{3}$ & Vegetable \\
\hline Carissa caranda & Karandas & Apocynaceae & $\mathrm{N}^{2} \mathrm{P}^{2}$ & Preservation \\
\hline Phoenix spp. & Wild date palm & Arecaceae & $\mathrm{N}^{2} \mathrm{P}^{3}$ & Fruit tree \\
\hline Asclepias curassavica & Milkweed & Asclepiadaceae & $\mathrm{N}^{2} \mathrm{P}^{2}$ & Fibre \\
\hline Calendula officinalis & Marigold & Asteraceae & $\mathrm{N}^{3} \mathrm{P}^{3}$ & Ornamental \\
\hline Cardus onopardiodes & Musk thistles & Asteraceae & $\mathrm{N}^{2} \mathrm{P}^{2}$ & - \\
\hline Chrysanthemum spp. & Chrysanthemum & Asteraceae & $\mathrm{N}^{3} \mathrm{P}^{3}$ & Ornamental \\
\hline Helichrysum arenarium & Paper flower & Asteraceae & $\mathrm{N}^{2} \mathrm{P}^{3}$ & Ornamental \\
\hline Taraxacum officinale & Dandelion & Asteraceae & $\mathrm{N}^{1} \mathrm{P}^{1}$ & Juicy Weed \\
\hline Tussilago farfara & Coltsfoot & Asteraceae & $\mathrm{N}^{3} \mathrm{P}^{3}$ & Medicinal \\
\hline Berberis aristata & Berberry & Berberidaceae & $\mathrm{N}^{2} \mathrm{P}^{2}$ & Fruit, Dye, Medicinal \\
\hline Berberis lycium & Raisin Berberry & Berberidaceae & $\mathrm{N}^{2} \mathrm{P}^{2}$ & Fruit, Medicinal \\
\hline Campsis grandiflora & Trumpet vine & Bignoniaceae & $\mathrm{N}^{2} \mathrm{P}^{3}$ & Ornamental \\
\hline Jacaranda mimosifolia & Jacaranda & Bignoniaceae & $\mathrm{N}^{3} \mathrm{P}^{3}$ & Ornamental \\
\hline Brassica campestris & Mustard & Brassicaceae & $\mathrm{N}^{1} \mathrm{P}^{1}$ & Oilseed crop \\
\hline Opuntia spp. & Prickly pear & Cactaceae & $\mathrm{N}^{2} \mathrm{P}^{2}$ & - \\
\hline Crataeva religiosa & Barna & Cannabinaceae & $\mathrm{N}^{2} \mathrm{P}^{3}$ & - \\
\hline Dianthus caryophyllus & Carnation & Caprifoliaceae & $\mathrm{N}^{3} \mathrm{P}^{3}$ & Ornamental \\
\hline Carica papaya & Papaya & Caricaceae & $\mathrm{N}^{1} \mathrm{P}^{1}$ & Fruit edible \\
\hline Chenopodium album & White goosefoot & Chenopodiaceae & $\mathrm{N}^{3} \mathrm{P}^{3}$ & Fodder \\
\hline Terminalia spp. & Myrobalan & Combretaceae & $\mathrm{N}^{1} \mathrm{P}^{1}$ & $\begin{array}{l}\text { Tree, Timber, Fruit, Medicinal, Fuel, Bark, for tanning, } \\
\text { Dyeing }\end{array}$ \\
\hline Cucurbita pepo & Pumpkin & Cucurbitaceae & $\mathrm{N}^{1} \mathrm{P}^{1}$ & Vegetable \\
\hline Emblica officinalis & Indian gooseberry & Euphorbiaceae & $\mathrm{N}^{2} \mathrm{P}^{2}$ & Medicinal \\
\hline Euphorbia royleana & Euphorbia & Euphorbiaceae & $\mathrm{N}^{3} \mathrm{P}^{3}$ & Juicy \\
\hline Ricinus cummunis & Caster oil plant & Euphorbiaceae & $\mathrm{P}^{2}$ & Oilseed, Softwooded \\
\hline Acacia arabica & Acacia & Fabaceae & $\mathrm{N}^{2} \mathrm{P}^{2}$ & $\begin{array}{c}\text { Fodder, Medicinal, Tanning industry, Dyeing, } \\
\text { Ornamental }\end{array}$ \\
\hline Acacia catechu & Acacia & Fabaceae & $\mathrm{N}^{1} \mathrm{P}^{1}$ & Timber, Fodder, Fuel-wood \\
\hline Albizzia lebbek & Siris & Fabaceae & $\mathrm{N}^{3} \mathrm{P}^{3}$ & $\begin{array}{l}\text { Fodder, Timber, Fuel } \\
\end{array}$ \\
\hline Bauhinia vahlii & Camel's foot & Fabaceae & $\mathrm{N}^{2} \mathrm{P}^{3}$ & Fodder, Fuel, Vegetable \\
\hline Butea monosperma & Palas & Fabaceae & $\mathrm{N}^{3} \mathrm{P}^{3}$ & Fuel, Timber, Food for lac insect, Medicinal \\
\hline Cassia fistula & Indian laburnum & Fabaceae & $\mathrm{N}^{2} \mathrm{P}^{2}$ & Avenue tree \\
\hline Cajanus cajan & Pigeon pea & Fabaceae & $\mathrm{N}^{3} \mathrm{P}^{3}$ & Food \\
\hline Dalbergia sissoo & Sissoo & Fabaceae & $\mathrm{N}^{1} \mathrm{P}^{1}$ & Timber, Fuel, Fodder, Shade \\
\hline Delonix regia & Gulmohar & Fabaceae & $\mathrm{N}^{2} \mathrm{P}^{2}$ & Avenue tree \\
\hline Leucaena leucocephala & Ipil-ipil & Fabaceae & $\mathrm{N}^{3} \mathrm{P}^{1}$ & Fuel, Fodder \\
\hline Medicago sativa & Alfalfa & Fabaceae & $\mathrm{N}^{1} \mathrm{P}^{2}$ & Fodder \\
\hline Melilotus spp. & Mellilot & Fabaceae & $\mathrm{N}^{1} \mathrm{P}^{2}$ & Fodder \\
\hline Pisum sativum & Garden pea & Fabaceae & $\mathrm{N}^{3} \mathrm{P}^{3}$ & Vegetable \\
\hline Tamarindus indica & Tamarind & Fabaceae & $\mathrm{N}^{3} \mathrm{P}^{3}$ & Avenue tree, Condiment, Fruit, Fodder, Oil \\
\hline Trifolium alexandrinum & Egyptian clover & Fabaceae & $\mathrm{N}^{1} \mathrm{P}^{1}$ & $\begin{array}{l}\text { Fodder } \\
\end{array}$ \\
\hline Trifolium pretense & Red clover & Fabaceae & $\mathrm{N}^{1} \mathrm{P}^{2}$ & Fodder \\
\hline Trifolium repens & White clover & Fabaceae & $\mathrm{N}^{1} \mathrm{P}^{1}$ & Fodder, Cover crop \\
\hline Vicia spp. & Field beans & Fabaceae & $\mathrm{N}^{3} \mathrm{P}^{3}$ & Edible, Fodder \\
\hline Geranium spp. & Geranium & Geraniaceae & $\mathrm{N}^{3} \mathrm{P}^{3}$ & Ornamental \\
\hline Hypericum spp. & Hypericum & Hypericaceae & $\mathrm{P}^{1}$ & $\begin{array}{l}\text { Twigs used as toothbrush, seed as condiment for } \\
\text { flavouring curries }\end{array}$ \\
\hline Lilium spp. & Lilium & Liliaceae & $\mathrm{P}^{3}$ & Ornamental \\
\hline Woodfordia fruticosa & Dhawi & Lytheraceae & $\mathrm{N}^{1} \mathrm{P}^{1}$ & Dye, Medicinal, Fodder, Fuel \\
\hline Hibiscns rosa-sinensis & Chinese rose & Malvaceae & $\mathrm{N}^{2} \mathrm{P}^{2}$ & Ornamental \\
\hline Malvaviscns arboreus & Malvaviscus & Malvaceae & $\mathrm{N}^{2} \mathrm{P}^{2}$ & Ornamental \\
\hline Azadirachta indica & Mangosa & Meliaceae & $\mathrm{N}^{2} \mathrm{P}^{3}$ & Avenue tree, Forest tree \\
\hline Cedrella toona & Cedrella & Meliaceae & $\mathrm{N}^{1} \mathrm{P}^{2}$ & Woody, Furniture \\
\hline
\end{tabular}


International Journal of Science and Research (IJSR)

ISSN (Online): 2319-7064

Index Copernicus Value (2013): 6.14 | Impact Factor (2015): 6.391

\begin{tabular}{|c|c|c|c|c|}
\hline Callistemon citrinus & Bottle brush & Myrtaceae & $\mathrm{N}^{1} \mathrm{P}^{1}$ & Ornamental \\
\hline Eucalyptus camaldulensis & Eucalyptus & Myrtaceae & $\mathrm{N}^{1} \mathrm{P}^{1}$ & $\begin{array}{c}\text { Avenue tree, Fuel wood, Charcoal, Paper pulp, } \\
\text { Termite-resistant timber, Medicinal }\end{array}$ \\
\hline Psidium guajava & Guava & Myrtaceae & $\mathrm{N}^{1} \mathrm{P}^{1}$ & $\begin{array}{l}\text { Fruit } \\
\end{array}$ \\
\hline Syzygium cumini & Jambolan & Myrtaceae & $\mathrm{N}^{1} \mathrm{P}^{1}$ & Avenue tree, Fruit \\
\hline Argemone mexicana & Prickly poppy & Papaveraceae & $\mathrm{P}^{3}$ & - \\
\hline Papaver rhoeas & Poppy & Papaveraceae & $\mathrm{N}^{3} \mathrm{P}^{3}$ & Ornamental \\
\hline Plantago spp. & Plantago & Plantaginaceae & $\mathrm{N}^{2} \mathrm{P}^{1}$ & - \\
\hline Cynodon dactylon & Dubgrass & Poaceae & $\mathrm{P}^{3}$ & Fodder \\
\hline Sorghum vulgare & Sorghum & Poaceae & $\mathrm{P}^{1}$ & Grain, Fodder \\
\hline Grevillea robusta & Silky oak & Proteaceae & $\mathrm{N}^{1} \mathrm{P}^{1}$ & Avenue tree, Ornamental, Timber \\
\hline Punica granatum & Pomegranate & Punicaceae & $\mathrm{N}^{2} \mathrm{P}^{1}$ & $\begin{array}{c}\text { Fruit } \\
\end{array}$ \\
\hline Punica nana & Wild pomegranate & Punicaceae & $\mathrm{N}^{2} \mathrm{P}^{1}$ & Fruit \\
\hline Anemone spp. & Wood anemone & Rananculaceae & $\mathrm{P}^{3}$ & Ornamental \\
\hline Clematis spp. & Clematis & Ranunculaceae & $\mathrm{N}^{2} \mathrm{P}^{2}$ & Medicinal \\
\hline Delphinium roylei & Larkspur & Ranunculaceae & $\mathrm{N}^{3} \mathrm{P}^{3}$ & Ornamental \\
\hline Ranunculus arvensis & Buttercup. & Ranunculaceae & $\mathrm{N}^{3} \mathrm{P}^{3}$ & - \\
\hline Rosa macrophylla & Rose & Rosaceae & $\mathrm{N}^{3} \mathrm{P}^{1}$ & Ornamental \\
\hline Rosa moschata & Wild rose & Rosaceae & $\mathrm{N}^{3} \mathrm{P}^{1}$ & Ornamental \\
\hline Rubus spp. & Berries & Rosaceae & $\mathrm{N}^{2} \mathrm{P}^{2}$ & Fruit \\
\hline Antirrhinum majus & Antirrhinum & Scrophulariaceae & $\mathrm{N}^{2} \mathrm{P}^{2}$ & Ornamental \\
\hline Sapindus mukorosii & Soapnut & Sapindaceae & $\mathrm{N}^{1} \mathrm{P}^{1}$ & Avenue tree, Fruit used as soap, Timber \\
\hline Lycopersicum esculentum & Tomato & Solanaceae & $\mathrm{N}^{3} \mathrm{P}^{3}$ & Vegetable \\
\hline Grewia optiva & Beolor Bhemal & Tiliaceae $=$ & $\mathrm{N}^{1} \mathrm{P}^{1}$ & Fodder, Timber, Fruit \\
\hline Vitis vinifera & Grapes & Vitaceae & $\mathrm{N}^{3} \mathrm{P}^{3}$ & Fruit \\
\hline June & & $>$ & & - \\
\hline Adhatoda vasica & Basuti & Acanthaceae & $\mathrm{N}^{2} \mathrm{P}^{2}$ & - \\
\hline Amaranthus paniculatus & Amaranth & Agavaceae & $\mathrm{P}^{2}$ & - \\
\hline Allium cepa & Onion & Amaryllidaceae & $\mathrm{N}^{3} \mathrm{P}^{3}$ & Vegetable \\
\hline Coriandrum sativum & Coriander & Apiaceae & $\mathrm{N}^{3} \mathrm{P}^{3}$ & Condiment \\
\hline Phoenix spp. & Wild date palm & Arecaceae & $\mathrm{N}^{2} \mathrm{P}^{3}$ & Fruit tree \\
\hline Asclepias curassavica & Milkweed & Asclepiadaceae & $\mathrm{N}^{2} \mathrm{P}^{2}$ & Fibre \\
\hline Bidens pilosa & Badi gumbri & Asteraceae & $\mathrm{N}^{3} \mathrm{P}^{3}$ & - \\
\hline Calendula officinalis & Marigold & Asteraceae & $\mathrm{N}^{3} \mathrm{P}^{3}$ & Ornamental \\
\hline Cardus onopardiodes & Musk thistles & Asteraceae & $\mathrm{N}^{2} \mathrm{P}^{2}$ & - \\
\hline Chrysanthemum spp. & Chrysanthemum & Asteraceae & $\mathrm{N}^{3} \mathrm{P}^{3}$ & Ornamental \\
\hline Cichorium intybus & Chichory & Asteraceae & $\mathrm{N}^{3} \mathrm{P}^{3}$ & - \\
\hline Helichrysum arenarium & Paper flower & Asteraceae & $\mathrm{N}^{2} \mathrm{P}^{3}$ & Ornamental \\
\hline Senecio spp. & Ragwort & Asteraceae & $\mathrm{N}^{3} \mathrm{P}^{3}$ & (C) - \\
\hline Solidago longifolia & Golden rod & Asteraceae & $\mathrm{N}^{2} \mathrm{P}^{3}$ & Ornamental \\
\hline Sonchus spp. & Sow thistle & Asteraceae & $\mathrm{N}^{3} \mathrm{P}^{3}$ & 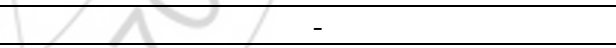 \\
\hline Taraxacum officinale & Dandelion & Asteraceae & $\mathrm{N}^{1} \mathrm{P}^{1}$ & Juicy weed \\
\hline Tussilago farfara & Coltsfoot & Asterceae & $\mathrm{N}^{3} \mathrm{P}^{3}$ & Medicinal \\
\hline Berberis aristata & Berberry & Berberidaceae & $\mathrm{N}^{2} \mathrm{P}^{2}$ & Fruit, Dye, Medicinal \\
\hline Berberis lyceum & Raisin berberry & Berberidaceae & $\mathrm{N}^{2} \mathrm{P}^{2}$ & $\begin{array}{ll}\text { Fruit, Medicinal } \\
\end{array}$ \\
\hline Campsis grandiflora & Trumpet vine & Bignoniaceae & $\mathrm{N}^{2} \mathrm{P}^{3}$ & Ornamental \\
\hline Jacaranda mimosifolia & Jacaranda & Bignoniaceae & $\mathrm{N}^{3} \mathrm{P}^{3}$ & Ornamental \\
\hline Cynoglossum spp. & Hounds tongue & Boraginaceae & $\mathrm{N}^{3} \mathrm{P}^{3}$ & - \\
\hline Cannabis sativa & Hemp & Cannabinaceae & $\mathrm{N}^{2} \mathrm{P}^{2}$ & Alkaloid Fibre \\
\hline Dianthus caryophyllus & Carnation & Caprifoliaceae & $\mathrm{N}^{3} \mathrm{P}^{3}$ & Ornamental \\
\hline Convolvulus arvensis & Convolvulus & Convolvulaceae & $\mathrm{N}^{3} \mathrm{P}^{3}$ & - \\
\hline Momordica charantia & Bitter gourd & Cucurbitaceae & $\mathrm{N}^{3} \mathrm{P}^{3}$ & Vegetable \\
\hline Hevea brasiliensis & Rubber tree & Euphorbiaceae & $\mathrm{N}^{1} \mathrm{P}^{3}$ & Ornamental \\
\hline Ricinus communis & Caster oil plant & Euphorbiaceae & $\mathrm{P}^{2}$ & Oilseed, Softwooded \\
\hline Acacia arabica & Acacia & Fabaceae & $\mathrm{N}^{2} \mathrm{P}^{2}$ & $\begin{array}{c}\text { Fodder, Medicinal, Tanning industry, Dyeing, } \\
\text { Ornamental }\end{array}$ \\
\hline Acacia catechu & Acacia & Fabaceae & $\mathrm{N}^{1} \mathrm{P}^{1}$ & Timber, Fodder, Fuel-wood \\
\hline Cassia fistula & Indian laburnum & Fabaceae & $\mathrm{N}^{2} \mathrm{P}^{2}$ & Avenue tree \\
\hline Cajanus cajan & Piegon pea & Fabaceae & $\mathrm{N}^{3} \mathrm{P}^{3}$ & Food \\
\hline Erythrina suberosa & Coral tree & Fabaceae & $\mathrm{N}^{2} \mathrm{P}^{2}$ & - \\
\hline Delonix regia & Gulmohar & Fabaceae & $\mathrm{N}^{2} \mathrm{P}^{2}$ & Avenue tree \\
\hline Indigofera spp. & Indigofera & Fabaceae & $\mathrm{N}^{2} \mathrm{P}^{2}$ & - \\
\hline Leucaena leucocephala & Ipil-ipil & Fabaceae & $\mathrm{N}^{3} \mathrm{P}^{1}$ & Fuel, Fodder \\
\hline Medicago sativa & Alfalfa & Fabaceae & $\mathrm{N}^{1} \mathrm{P}^{2}$ & Fodder \\
\hline Pisum sativum & Garden pea & Fabaceae & $\mathrm{N}^{3} \mathrm{P}^{3}$ & Vegetable \\
\hline Trifolium alexandrinum & Egyptian clover & Fabaceae & $\mathrm{N}^{1} \mathrm{P}^{1}$ & Fodder \\
\hline Trifolium pretense & Red clover & Fabaceae & $\mathrm{N}^{1} \mathrm{P}^{2}$ & Fodder \\
\hline
\end{tabular}




\section{International Journal of Science and Research (IJSR) ISSN (Online): 2319-7064}

Index Copernicus Value (2013): 6.14 | Impact Factor (2015): 6.391

\begin{tabular}{|c|c|c|c|c|}
\hline Trifoliumrepens & White clover & Fabaceae & $\mathrm{N}^{1} \mathrm{P}^{1}$ & Fodder, Cover crop \\
\hline Vicia spp. & Field beans & Fabaceae & $\mathrm{N}^{3} \mathrm{P}^{3}$ & Edible, Fodder \\
\hline Castanea sativa & Sweet chestnut & Fagaceae & $\mathrm{N}^{3} \mathrm{P}^{3}$ & Timber, Seed, Nuts, Edible \\
\hline Geranium spp. & Geranium & Geraniaceae & $\mathrm{N}^{3} \mathrm{P}^{3}$ & Ornamental \\
\hline Hypericum spp. & Hypericum & Hypericaceae & $\mathrm{P}^{1}$ & $\begin{array}{c}\text { Twigs used as toothbrush, seed as comdiment for } \\
\text { flavouring curries }\end{array}$ \\
\hline Ocimum basilicum & Basil & Lamiaceae & $\mathrm{N}^{2} \mathrm{P}^{3}$ & - \\
\hline Lilium spp. & Lilium & Liliaceae & $\mathrm{P}^{3}$ & Ornamental \\
\hline Althaea rosea & Hollyhock & Malvaceae & $\mathrm{N}^{2} \mathrm{P}^{2}$ & Ornamental \\
\hline Malva sylvestris & Mallow & Malvaceae & $\mathrm{N}^{2} \mathrm{P}^{3}$ & Ornamental \\
\hline Malvaviscus arboreus & Malvaviscus & Malvaceae & $\mathrm{N}^{2} \mathrm{P}^{2}$ & Ornamental \\
\hline Azadirachta indica & Mangosa & Meliaceae & $\mathrm{N}^{2} \mathrm{P}^{3}$ & Avenue tree, Forest tree \\
\hline Cedrela toona & Cedrella & Meliaceae & $\mathrm{N}^{1} \mathrm{P}^{2}$ & Woody, Furniture \\
\hline Musa sapientum & Banana & Musaceae & $\mathrm{N}^{2} \mathrm{P}^{3}$ & Fruit \\
\hline Callistemon citrinus & Bottle brush & Myrtaceae & $\mathrm{N}^{1} \mathrm{P}^{1}$ & Ornamental \\
\hline Eucalyptus camaldulensis & Eucalyptus & Myrtaceae & $\mathrm{N}^{1} \mathrm{P}^{1}$ & $\begin{array}{c}\text { Avenue tree, Fuel wood, Charcoal, Paper pulp, } \\
\text { Termite-resistant timber, Medicinal }\end{array}$ \\
\hline Psidium guajava & Guava & Myrtaceae & $\mathrm{N}^{1} \mathrm{P}^{1}$ & Fruit \\
\hline Syzygium cumini & Jambolan & Myrtaceae & $\mathrm{N}^{1} \mathrm{P}^{1}$ & Avenue tree, Fruit \\
\hline Argemone mexicana & Prickly poppy & Papaveraceae & $\mathrm{P}^{3}$ & - \\
\hline Eschscholzia californica & Californian poppy & Papaveraceae & $\mathrm{N}^{3} \mathrm{P}^{1}$ & Ornamental \\
\hline Plantago spp. & Plantago & Plantaginaceae & $\mathrm{N}^{2} \mathrm{P}^{1}$ & - \\
\hline Cynodon dactylon & Dubgrass & Poaceae & $\mathrm{P}^{3}$ & Fodder \\
\hline Sorghum vulgare & Sorgum & Poaceae & $\mathrm{P}^{1}$ & Grain, Fodder \\
\hline Polygonum spp. & Polygonum & Polygonaceae & $\mathrm{N}^{3} \mathrm{P}^{3}$ & - \\
\hline Rumex spp. & Rumex & Polygonaceae & $\mathrm{N}^{3} \mathrm{P}^{3}$ & - \\
\hline Portulaca grandiflora & Portulaca & Portulacaceae & $\mathrm{N}^{2} \mathrm{P}^{1}$ & Ornamental \\
\hline Anemone spp. & Wood anemone & Ranunculaceae & $\mathrm{P}^{3}$ & Ornamental \\
\hline Ranunculus arvensis & Butter cup & Ranunculaceae & $\mathrm{N}^{3} \mathrm{P}^{3}$ & - \\
\hline Rosa moschata & Wild rose & Rosaceae & $\mathrm{N}^{3} \mathrm{P}^{1}$ & Ornamental \\
\hline Rubus spp. & Berries & Rosaceae & $\mathrm{N}^{2} \mathrm{P}^{2}$ & Fruit \\
\hline Antirrhinum majus & Antirrhinum & Scrophulariaceae & $\mathrm{N}^{2} \mathrm{P}^{2}$ & Ornamental \\
\hline Sapindus mukorosii & Soapnut & Sapindaceae & $\mathrm{N}^{1} \mathrm{P}^{1}$ & Avenue tree, Fruit used as soap, Timber \\
\hline Lycopersicum esculentum & Tomato & Solanaceae & $\mathrm{N}^{3} \mathrm{P}^{3}$ & Vegetable \\
\hline Solanum melongena & Brinjal & Solanaceae & $\mathrm{N}^{3} \mathrm{P}^{3}$ & Ornamental \\
\hline Grewia optiva & Beolor Bhemal & Tiliaceae & $\mathrm{N}^{1} \mathrm{P}^{1}$ & Fodder, Timber, Fruit \\
\hline Tilia spp. & Lime basswood & Tiliaceae & $\mathrm{N}^{1}$ & Avenue tree, Ornamental, Timber \\
\hline Vitex negundo & Indian privet & Verbenaceae & $\mathrm{N}^{1} \mathrm{P}^{2}$ & Insect repellent, Medicinal \\
\hline Vitis vinifera & Grapes & Vitaceae & $\mathrm{N}^{3} \mathrm{P}^{3}$ & Fruit \\
\hline July & & & & - \\
\hline Adhatoda vasica & Basuti & Acanthaceae & $\mathrm{N}^{2} \mathrm{P}^{2}$ & +2 \\
\hline Rungia parviflora & Rungia & Acanthaceae & $\mathrm{N}^{3} \mathrm{P}^{3}$ & 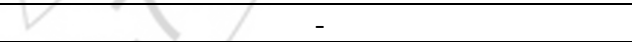 \\
\hline Amaranthus paniculatus & Amaranth & Agavaceae & $\mathrm{P}^{2}$ & +2 \\
\hline Phoenix spp. & Wild date palm & Arecaceae & $\mathrm{N}^{2} \mathrm{P}^{3}$ & Fruit tree \\
\hline Ageratum conyzoides & $\begin{array}{c}\text { Ageratum or Goat } \\
\text { weed }\end{array}$ & Asteraceae & $\mathrm{N}^{3} \mathrm{P}^{3}$ & Ornamental \\
\hline Aster spp. & Star wort & Asteraceae & $\mathrm{N}^{3} \mathrm{P}^{3}$ & Ornamental \\
\hline Bidens pilosa & Badi-gumbri & Asteraceae & $\mathrm{N}^{3} \mathrm{P}^{3}$ & - \\
\hline Calendula officinalis & Marigold & Asteraceae & $\mathrm{N}^{3} \mathrm{P}^{3}$ & Ornamental \\
\hline Cardus onopardiodes & Musk thistles & Asteraceae & $\mathrm{N}^{2} \mathrm{P}^{2}$ & - \\
\hline Chrysanthemum spp. & Chrysanthemum & Asteraceae & $\mathrm{N}^{3} \mathrm{P}^{3}$ & Ornamental \\
\hline Dahlia pinnata & Dahlia & Asteraceae & $\mathrm{N}^{2} \mathrm{P}^{2}$ & Ornamental \\
\hline Eupatorium spp. & Throughwort & Asteraceae & $\mathrm{N}^{3} \mathrm{P}^{3}$ & - \\
\hline Helianthus annuus & Sunflower & Asteraceae & $\mathrm{N}^{1} \mathrm{P}^{1}$ & Oilseed, Ornamental, Fodder \\
\hline Senecio spp. & Ragwrot & Asteraceae & $\mathrm{N}^{3} \mathrm{P}^{3}$ & - \\
\hline Solidago longifolia & Golden rod & Asteraceae & $\mathrm{N}^{2} \mathrm{P}^{3}$ & Ornamental \\
\hline Sonchus spp. & Sow thistle & Asteraceae & $\mathrm{N}^{3} \mathrm{P}^{3}$ & - \\
\hline Tagetus erectus & Marigold & Asteraceae & $\mathrm{N}^{3} \mathrm{P}^{3}$ & Aromatic, Ornamental \\
\hline Taraxacum officinale & Dandelion & Asteraceae & $\mathrm{N}^{1} \mathrm{P}^{1}$ & Juicy Weed \\
\hline Impatiens balsamina & Pink balsam & Balsaminaceae & $\mathrm{N}^{1} \mathrm{P}^{2}$ & Medicinal, Aromatic \\
\hline Impatiens glandulifera & Balsam & Balsaminaceae & $\mathrm{N}^{2} \mathrm{P}^{2}$ & Medicinal \\
\hline Campsis grandiflora & Trumpetvine & Bignoniaceae & $\mathrm{N}^{2} \mathrm{P}^{3}$ & Ornamental \\
\hline Convolvulus arvensis & Convolvulus & Convolvulaceae & $\mathrm{N}^{3} \mathrm{P}^{3}$ & - \\
\hline Cuscuta reflexa & Amarbel & Convolvulaceae & $\mathrm{P}^{3}$ & Parasitic, Succulent, Medicinal \\
\hline Cucumis spp. & Cucumber & Cucurbitaceae & $\mathrm{N}^{1} \mathrm{P}^{1}$ & Edible \\
\hline Luffa cylindrical & Ridged gourd & Cucurbitaceae & $\mathrm{N}^{3} \mathrm{P}^{3}$ & - \\
\hline Momordica charantia & Bitter gourd & Cucurbitaceae & $\mathrm{N}^{3} \mathrm{P}^{3}$ & Vegetable \\
\hline
\end{tabular}




\section{International Journal of Science and Research (IJSR) \\ ISSN (Online): 2319-7064}

Index Copernicus Value (2013): 6.14 | Impact Factor (2015): 6.391

\begin{tabular}{|c|c|c|c|c|}
\hline Hevea brasiliensis & Rubber tree & Euphorbiaceae & $\mathrm{N}^{1} \mathrm{P}^{3}$ & Ornamental \\
\hline Ricinus communis & Caster oil plant & Euphorbiaceae & $\mathrm{P}^{2}$ & Oilseed, Softwooded \\
\hline Acacia Arabica & Acacia & Fabaceae & $\mathrm{N}^{2} \mathrm{P}^{2}$ & $\begin{array}{l}\text { Fodder, Medicinal, Tanning industry, Dyeing, } \\
\text { Ornamental }\end{array}$ \\
\hline Acacia catechu & Acacia & Fabaceae & $\mathrm{N}^{1} \mathrm{P}^{1}$ & Timber, Fodder, Fuel-wood \\
\hline Butea monosperma & Palas & Fabaceae & $\mathrm{N}^{3} \mathrm{P}^{3}$ & Fuel, Timber, Food for lac insect, Medicinal \\
\hline Cassia fistula & Indian laburnum & Fabaceae & $\mathrm{N}^{2} \mathrm{P}^{2}$ & Avenue tree \\
\hline Cajanus cajan & Pigeon pea & Fabaceae & $\mathrm{N}^{3} \mathrm{P}^{3}$ & Food \\
\hline Glycine max & Soyabean & Fabaceae & $\mathrm{N}^{3} \mathrm{P}^{3}$ & Oilseed crop \\
\hline Indigofera spp. & Indigofera & Fabaceae & $\mathrm{N}^{2} \mathrm{P}^{2}$ & - \\
\hline Leucaena leucocephala & Ipil-ipil & Fabaceae & $\mathrm{N}^{3} \mathrm{P}^{1}$ & Fuel, Fodder \\
\hline Medicago sativa & Alfalfa & Fabaceae & $\mathrm{N}^{1} \mathrm{P}^{2}$ & Fodder \\
\hline Melilotus spp. & Mellilot & Fabaceae & $\mathrm{N}^{1} \mathrm{P}^{2}$ & Fodder \\
\hline Pisum sativum & Garden pea & Fabaceae & $\mathrm{N}^{3} \mathrm{P}^{3}$ & Vegetable \\
\hline Trifolium alexandrinum & Egyptian clover & Fabaceae & $\mathrm{N}^{1} \mathrm{P}^{\mathrm{I}}$ & Fodder \\
\hline Trifolium pretense & Red clover & Fabaceae & $\mathrm{N}^{1} \mathrm{P}^{2}$ & Fodder \\
\hline Trifolium repens & White clover & Fabaceae & $\mathrm{N}^{1} \mathrm{P}^{\mathrm{I}}$ & Fodder, Cover crop \\
\hline Vicia spp. & Field beans & Fabaceae & $\mathrm{N}^{3} \mathrm{P}^{3}$ & Edible, Fodder \\
\hline Castanea sativa & Sweet chestnut & Fagaceae & $\mathrm{N}^{3} \mathrm{P}^{3}$ & Timber, Seed, Nuts, Edible \\
\hline Geranium spp. & Geranium & Geraniaceae & $\mathrm{N}^{3} \mathrm{P}^{3}$ & Ornamental \\
\hline Hypericum spp. & Hypericum & Hypericaceae & $\mathrm{P}^{1}$ & $\begin{array}{l}\text { Twigs used as toothbrush, seed as condiment for } \\
\text { flavouring curries }\end{array}$ \\
\hline Mentha viridis & Mint & Lamiaceae & $\mathrm{N}^{3}$ & Aromatic herb \\
\hline Ocimum basilicum & Basil & Lamiaceae & $\mathrm{N}^{2} \mathrm{P}^{3}$ & - \\
\hline Origanum vulgare & Marjoram & Lamiaceae & $\mathrm{N}^{2} \mathrm{P}^{2}$ & Aromatic herb, Oil \\
\hline Salvia spp. & Red sage & Lamiaceae & $\mathrm{N}^{2} \mathrm{P}^{2}$ & Medicinal \\
\hline Stachys spp. & Woundwort & Lamiaceae & $\mathrm{N}^{2} \mathrm{P}^{3}$ & - \\
\hline Lagerstroemia indica & Pride of india & Lytheraceae & $\mathrm{N}^{2} \mathrm{P}^{2}$ & Timber, Ornamental \\
\hline Abelmoschus esculentus & Lady's finger & Malvaceae & $\mathrm{N}^{2} \mathrm{P}^{2}$ & Vegetable \\
\hline Althaea rosea & Holly hock & Malvaceae & $\mathrm{N}^{2} \mathrm{P}^{2}$ & Ornamental \\
\hline Gossypium arboretum & Cotton & Malvaceae & $\mathrm{N}^{1} \mathrm{P}^{2}$ & - \\
\hline Malva sylvestris & Mallow & Malvaceae & $\mathrm{N}^{2} \mathrm{P}^{3}$ & Ornamental \\
\hline Malvaviscus arboreus & Malvaviscus & Malvaceae & $\mathrm{N}^{2} \mathrm{P}^{2}$ & Ornamental \\
\hline Azadirachta indica & Mangosa & Meliaceae & $\mathrm{N}^{2} \mathrm{P}^{3}$ & Avenue tree, Forest tree \\
\hline Musa sapientum & Banana & Musaceae & $\mathrm{N}^{2} \mathrm{P}^{3}$ & Fruit \\
\hline Callistemon citrinus & Bottle brush & Myrtaceae & $\mathrm{N}^{1} \mathrm{P}^{1}$ & Ornamental \\
\hline Epilobium spp. & Willow herb & Ongraceae & $\mathrm{N}^{2} \mathrm{P}^{2}$ & $e^{2}-$ \\
\hline Argemone Mexicana & Prickly poppy & Papaveraceae & $\mathrm{P}^{3}$ & 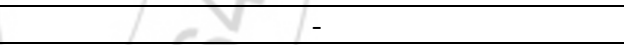 \\
\hline Sesamum indicum & Sesamum & Pedaliaceae & $\mathrm{N}^{1} \mathrm{P}^{1}$ & Oilseed \\
\hline Cynodon dactylon & Dubgrass & Poaceae & $\mathrm{P}^{3}$ & Fodder \\
\hline Zea mays & Maize & Poaceae & $\mathrm{P}^{1}$ & Fodder \\
\hline Fagopyrum sagittatum & Buck wheat & Polygonaceae & $\mathrm{N}^{1} \mathrm{P}^{2}$ & Grain, Fodder \\
\hline Polygonum spp. & Polygonum & Polygonaceae & $\mathrm{N}^{3} \mathrm{P}^{3}$ & 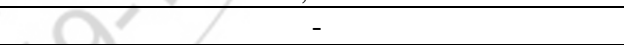 \\
\hline Rumex spp. & Rumex & Polygonaceae & $\mathrm{N}^{3} \mathrm{P}^{3}$ & $x_{-3}$ \\
\hline Portulaca grandiflora & Portulaca & Portulacaceae & $\mathrm{N}^{2} \mathrm{P}^{1}$ & Ornamental \\
\hline Zizyphus jujuba & Chinese date & Rhamnaceae & $\mathrm{N}^{1} \mathrm{P}^{3}$ & Fruit, Fodder, Oilseed \\
\hline Eriobotrya japonica & Loquat & Rosaceae & $\mathrm{N}^{1} \mathrm{P}^{1}$ & Fruit \\
\hline Capsicum spp. & Chillies & Solanaceae & $\mathrm{N}^{3} \mathrm{P}^{3}$ & Vegetable \\
\hline Datura stramonium & Thorn apple & Solanaceae & $\mathrm{P}^{3}$ & Medicinal \\
\hline Lycopersicum esculentum & Tomato & Solanaceae & $\mathrm{N}^{3} \mathrm{P}^{3}$ & Vegetable \\
\hline Solanum melongena & Brinjal & Solanaceae & $\mathrm{N}^{3} \mathrm{P}^{3}$ & Vegetable \\
\hline Grewia optiva & Beolor Bhemal & Tiliaceae & $\mathrm{N}^{1} \mathrm{P}^{\mathrm{I}}$ & Fodder, Timber, Fruit \\
\hline \multicolumn{5}{|l|}{ August } \\
\hline Adhatoda vasica & Basuti & Acanthaceae & $\mathrm{N}^{2} \mathrm{P}^{2}$ & - \\
\hline Rungia parviflora & Rungia & Acanthaceae & $\mathrm{N}^{3} \mathrm{P}^{3}$ & - \\
\hline Foeniculum vulgare & Funnel & Apiaceae & $\mathrm{N}^{3} \mathrm{P}^{3}$ & Condiment \\
\hline Ageratum conyzoides & $\begin{array}{l}\text { Ageratum or Goat } \\
\text { weed }\end{array}$ & Asteraceae & $\mathrm{N}^{3} \mathrm{P}^{3}$ & Ornamental \\
\hline Aster spp. & Starwort & Asteraceae & $\mathrm{N}^{3} \mathrm{P}^{3}$ & Ornamental \\
\hline Bidens pilosa & Badi gumbri & Asteraceae & $\mathrm{N}^{3} \mathrm{P}^{3}$ & - \\
\hline Eupatorium spp. & Throughwort & Asteraceae & $\mathrm{N}^{3} \mathrm{P}^{3}$ & - \\
\hline Helianthus annuus & Sunflower & Asteraceae & $\mathrm{N}^{1} \mathrm{P}^{1}$ & Oilseed, Ornamental, Fodder \\
\hline Senecio spp. & Rajwort & Asteraceae & $\mathrm{N}^{3} \mathrm{P}^{3}$ & - \\
\hline Solidago longifolia & Golden rod & Asteraceae & $\mathrm{N}^{2} \mathrm{P}^{3}$ & Ornamental \\
\hline Sonchus spp. & Sow thistle & Asteraceae & $\mathrm{N}^{3} \mathrm{P}^{3}$ & - \\
\hline Tagetus erectus & Marigold & Asteraceae & $\mathrm{N}^{3} \mathrm{P}^{3}$ & Ornamental \\
\hline Taraxacum officinale & Dandelion & Asteraceae & $\mathrm{N}^{1} \mathrm{P}^{1}$ & Juicy Weed \\
\hline
\end{tabular}




\section{International Journal of Science and Research (IJSR) ISSN (Online): 2319-7064}

Index Copernicus Value (2013): 6.14 | Impact Factor (2015): 6.391

\begin{tabular}{|c|c|c|c|c|}
\hline Zinnia elegans & Zinnia & Asteraceae & $\mathrm{N}^{2} \mathrm{P}^{2}$ & Ornamental \\
\hline Impatiens balsamina & Pink balsam & Asteraceae & $\mathrm{N}^{1} \mathrm{P}^{2}$ & Medicinal \\
\hline Cannabis sativa & Hemp & Cannabinaceae & $\mathrm{N}^{2} \mathrm{P}^{2}$ & Alkaloid fibre \\
\hline Lonicera & Honey suckle & Caprifoliaceae & $\mathrm{N}^{3} \mathrm{P}^{3}$ & - \\
\hline Chenopodium album & White goose foot & Caprifoliaceae & $\mathrm{N}^{3} \mathrm{P}^{3}$ & Fodder \\
\hline Convolvulus arvensis & Convolvulus & Convolvulaceae & $\mathrm{N}^{3} \mathrm{P}^{3}$ & - \\
\hline Ipomoea batatas & Sweet potato & Convolvulaceae & $\mathrm{N}^{2} \mathrm{P}^{3}$ & Vegetable \\
\hline Ipomoea pulchella & Railway creeper & Convolvulaceae & $\mathrm{N}^{3} \mathrm{P}^{3}$ & - \\
\hline Cuscuta reflexa & Amarbel & Convolvulaceae & $\mathrm{P}^{3}$ & Medicinal \\
\hline Luffa cylindrical & Ridged gourd & Cucurbitaceae & $\mathrm{N}^{3} \mathrm{P}^{3}$ & - \\
\hline Momordica charantia & Bitter gourd & Cucurbitaceae & $\mathrm{N}^{3} \mathrm{P}^{3}$ & Vegetable \\
\hline Cucumis spp. & Cucumber & Cucurbitaceae & $\mathrm{N}^{1} \mathrm{P}^{1}$ & Edible \\
\hline Ricinus communis & Caster oil plant & Euphorbiaceae & $\mathrm{P}^{2}$ & Oilseed, Softwooded \\
\hline Butea monosperma & Palas & Fabaceae & $\mathrm{N}^{3} \mathrm{P}^{3}$ & Fuel, Timber, Food for lac insect, Medicinal \\
\hline Cajanus cajan & Pigeon Pea & Fabaceae & $\mathrm{N}^{3} \mathrm{P}^{3}$ & Food \\
\hline Glycine $\max$ & Soyabean & Fabaceae & $\mathrm{N}^{3} \mathrm{P}^{3}$ & Oilseed crop \\
\hline Indigofera spp. & Indigofera & Fabaceae & $\mathrm{N}^{2} \mathrm{P}^{2}$ & - \\
\hline Leucaena leucocephala & Ipil-ipil & Fabaceae & $\mathrm{N}^{3} \mathrm{P}^{1}$ & Fuel, Fodder \\
\hline Medicago sativa & Alfalfa & Fabaceae & $\mathrm{N}^{1} \mathrm{P}^{2}$ & Fodder \\
\hline Vicia spp. & Field beans & Fabaceae & $\mathrm{N}^{3} \mathrm{P}^{3}$ & Edible, Fodder \\
\hline Mentha viridis & Mint & Lamiaceae & $\mathrm{N}^{3}$ & Aromatic herb \\
\hline Ocimum basilicum & Basil & Lamiaceae & $\mathrm{N}^{2} \mathrm{P}^{3}$ & - \\
\hline Origanum vulgare & Marjoram & Lamiaceae & $\mathrm{N}^{2} \mathrm{P}^{2}$ & Oil \\
\hline Salvia spp. & Red sage & Lamiaceae & $\mathrm{N}^{2} \mathrm{P}^{2}$ & Medicinal \\
\hline Stachys spp. & Woundwort & Lamiaceae & $\mathrm{N}^{2} \mathrm{P}^{3}$ & $\sqrt{x}$ \\
\hline Asphodelus tenuifolius & Piazi & Liliaceae & $\mathrm{N}^{3} \mathrm{P}^{2}$ & - \\
\hline Lagerstroemia indica & Pride of india & Lytheraceae & $\mathrm{N}^{2} \mathrm{P}^{2}$ & Timber, Ornamental \\
\hline Abelmoschus esculentus & Lady's finger & Malvaceae & $\mathrm{N}^{2} \mathrm{P}^{2}$ & Vegetable \\
\hline Althaea rosea & Hollyhock & Malvaceae & $\mathrm{N}^{2} \mathrm{P}^{2}$ & Vegetable \\
\hline Gossypium arboreum & Cotton & Malvaceae & $\mathrm{N}^{1} \mathrm{P}^{2}$ & - \\
\hline Malva sylvestris & Mallow & Malvaceae & $\mathrm{N}^{2} \mathrm{P}^{3}$ & Ornamental \\
\hline Malvaviscus arboreus & Malvaviscus & Malvaceae & $\mathrm{N}^{2} \mathrm{P}^{2}$ & Ornamental \\
\hline Azadirachta indica & Mangosa & Meliaceae & $\mathrm{N}^{2} \mathrm{P}^{3}$ & Avenue tree, Forest tree \\
\hline Musa sapientum & Banana & Musaceae & $\mathrm{N}^{2} \mathrm{P}^{3}$ & Fruit \\
\hline Sesamum indicum & Sesamum & Pedaliaceae & $\mathrm{N}^{1} \mathrm{P}^{1}$ & Oilseed \\
\hline Plantago spp. & Plantago & Plantaginaceae & $\mathrm{N}^{2} \mathrm{P}^{1}$ & - \\
\hline Cynodon dactylon & Dubgrass & Poaceae & $\mathrm{P}^{3}$ & Fodder \\
\hline Zea mays & Maize & Poaceae & $\mathrm{P}^{1}$ & Fodder \\
\hline Fagopyrum sagittatum & Buck wheat & Polygonaceae & $\mathrm{N}^{1} \mathrm{P}^{2}$ & Grain, Fodder \\
\hline Polygonum spp. & Polygonum & Polygonaceae & $\mathrm{N}^{3} \mathrm{P}^{3}$ & - \\
\hline Rumex spp. & Rumex & Polygonaceae & $\mathrm{N}^{3} \mathrm{P}^{3}$ & 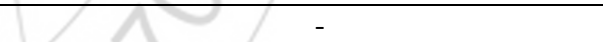 \\
\hline Portulaca grandiflora & Portulaca & Portulacaceae & $\mathrm{N}^{2} \mathrm{P}^{1}$ & Ornamental \\
\hline Zizyphus jujuba & Chinese date & Rhamnaceae & $\mathrm{N}^{1} \mathrm{P}^{3}$ & Fruit, Fodder, Oilseed \\
\hline Scrophularia spp. & Fig figwort & Scrophulariaceae & $\mathrm{N}^{1} \mathrm{P}^{2}$ & - \\
\hline Capsicum spp. & Chillies & Solanaceae & $\mathrm{N}^{3} \mathrm{P}^{3}$ & Vegetable \\
\hline Datura stramonium & Thorn apple & Solanaceae & $\mathrm{P}^{3}$ & Medicinal \\
\hline Lycopersicum esculentum & Tomato & Solanaceae & $\mathrm{N}^{3} \mathrm{P}^{3}$ & Vegetable \\
\hline Solanum melongena & Brinjal & Solanaceae & $\mathrm{N}^{3} \mathrm{P}^{3}$ & Vegetable \\
\hline \multicolumn{5}{|l|}{ September } \\
\hline Adhatoda vasica & Basuti & Acanthaceae & $\mathrm{N}^{2} \mathrm{P}^{2}$ & - \\
\hline Agave americana & Century plant & Agavaceae & $\mathrm{N}^{3} \mathrm{P}^{3}$ & - \\
\hline Foeniculum vulgare & Funnel & Apiaceae & $\mathrm{N}^{3} \mathrm{P}^{3}$ & Condiment \\
\hline Ageratum conyzoides & $\begin{array}{c}\text { Ageratum or Goat } \\
\text { weed }\end{array}$ & Asteraceae & $\mathrm{N}^{3} \mathrm{P}^{3}$ & Ornamental \\
\hline Aster spp. & Star-wort & Asteraceae & $\mathrm{N}^{3} \mathrm{P}^{3}$ & Ornamental \\
\hline Bidens pilosa & Badi- gumbri & Asteraceae & $\mathrm{N}^{3} \mathrm{P}^{3}$ & - \\
\hline Senecio spp. & Ragwort & Asteraceae & $\mathrm{N}^{3} \mathrm{P}^{3}$ & - \\
\hline Solidago longifolia & Golden rod & Asteraceae & $\mathrm{N}^{2} \mathrm{P}^{3}$ & Ornamental \\
\hline Sonchus spp. & Sow thistle & Asteraceae & $\mathrm{N}^{3} \mathrm{P}^{3}$ & - \\
\hline Tagetus erectus & Marigold & Asteraceae & $\mathrm{N}^{3} \mathrm{P}^{3}$ & Ornamental \\
\hline Taraxacum officinale & Dandelion & Asteraceae & $\mathrm{N}^{1} \mathrm{P}^{1}$ & Juicy Weed \\
\hline Zinnia elegans & Zinnia & Asteraceae & $\mathrm{N}^{2} \mathrm{P}^{2}$ & Ornamental \\
\hline Impatiens balsamina & Pink balsam & Balsaminaceae & $\mathrm{N}^{1} \mathrm{P}^{2}$ & Medicinal \\
\hline Impatiens glandulifera & Balsam & Balsaminaceae & $\mathrm{N}^{2} \mathrm{P}^{2}$ & Medicinal \\
\hline Cynoglossum spp. & Hounds tongue & Boraginaceae & $\mathrm{N}^{3} \mathrm{P}^{3}$ & - \\
\hline Brassica campestris & Sarson & Brassicaceae & $\mathrm{N}^{1} \mathrm{P}^{1}$ & Oilseed crop \\
\hline Cannabis sativa & Hemp & Cannabinaceae & $\mathrm{N}^{2} \mathrm{P}^{2}$ & Alkaloid fibre \\
\hline
\end{tabular}


International Journal of Science and Research (IJSR)

ISSN (Online): 2319-7064

Index Copernicus Value (2013): 6.14 | Impact Factor (2015): 6.391

\begin{tabular}{|c|c|c|c|c|}
\hline Convolvulus arvensis & Convolvulus & Convolvulaceae & $\mathrm{N}^{3} \mathrm{P}^{3}$ & - \\
\hline Ipomoea batatas & Sweet potato & Convolvulaceae & $\mathrm{N}^{2} \mathrm{P}^{3}$ & Vegetable \\
\hline Momordica charantia & Bitter gourd & cucurbitaceae & $\mathrm{N}^{3} \mathrm{P}^{3}$ & $\begin{array}{c}\text { Vegetable } \\
\end{array}$ \\
\hline Butea monosperma & Palas & Fabaceae & $\mathrm{N}^{3} \mathrm{P}^{3}$ & Fuel, Timber, Food for lac insect, Medicinal \\
\hline Cajanus cajan & Pigeon pea & Fabaceae & $\mathrm{N}^{3} \mathrm{P}^{3}$ & $\begin{array}{l}\text { Food } \\
\end{array}$ \\
\hline Leucaena leucocephala & Ipil-ipil & Fabaceae & $\mathrm{N}^{1} \mathrm{P}^{1}$ & Timber, Fuel, Fodder, Shade \\
\hline Hypericum spp. & Hypericum & Hypericaceae & $\mathrm{P}^{1}$ & $\begin{array}{l}\text { Twigs used as toothbrush, seed as condiment for } \\
\text { flavouring curries }\end{array}$ \\
\hline Mentha viridis & Mint & Lamiaceae & $\mathrm{N}^{3}$ & Aromatic herb \\
\hline Ocimum basilicum & Basil & Lamiaceae & $\mathrm{N}^{2} \mathrm{P}^{3}$ & - \\
\hline Asphodelus tenuifolius & Piazi & Liliaceae & $\mathrm{N}^{3} \mathrm{P}^{2}$ & - \\
\hline Lagerstroemia indica & Pride of india & Lytheraceae & $\mathrm{N}^{2} \mathrm{P}^{2}$ & Timber, Ornamental \\
\hline Abelmoschus esculentus & Lady's finger & Malvaceae & $\mathrm{N}^{2} \mathrm{P}^{2}$ & Vegetable \\
\hline Althaea rosea & Holly hock & Malvaceae & $\mathrm{N}^{2} \mathrm{P}^{2}$ & Ornamental \\
\hline Gossypium arboreum & Cotton & Malvaceae & $\mathrm{N}^{1} \mathrm{P}^{2}$ & - \\
\hline Malvaviscus arboreus & Malvaviscus & Malvaceae & $\mathrm{N}^{2} \mathrm{P}^{2}$ & Ornamental \\
\hline Azadirachta indica & Mangosa & Meliaceae & $\mathrm{N}^{2} \mathrm{P}^{3}$ & Avenue tree, Forest tree \\
\hline Musa sapientum & Banana & Musaceae & $\mathrm{N}^{2} \mathrm{P}^{3}$ & Fruit \\
\hline Callistemon citrinus & Bottle brush & Myrtaceae & $\mathrm{N}^{1} \mathrm{P}^{1}$ & Ornamental \\
\hline Epilobium spp. & Willow herb & Ongraceae & $\mathrm{N}^{2} \mathrm{P}^{2}$ & - \\
\hline Sesamum indicum & Sesasum & Pedaliaceae & $\mathrm{N}^{1} \mathrm{P}^{1}$ & Oilseed \\
\hline Plantago dactylon & Plantago & Plantiginaceae & $\mathrm{N}^{2} \mathrm{P}^{1}$ & - \\
\hline Cynodon dactylon & Dubgrass & Poaceae & $\mathrm{P}^{3}$ & Fodder \\
\hline Fagopyrum sagittatum & Buck wheat & Polygonaceae & $\mathrm{N}^{1} \mathrm{P}^{2}$ & Fodder \\
\hline Polygonum spp. & Polygonum & Polygonaceae & $\mathrm{N}^{3} \mathrm{P}^{3}$ & $\sqrt{36}$ \\
\hline Rumex spp. & Rumex & Polygonaceae & $\mathrm{N}^{3} \mathrm{P}^{3}$ & 6 \\
\hline Portulaca grandiflora & Portulaca & Portulacaceae & $\mathrm{N}^{2} \mathrm{P}^{1}$ & Ornamental \\
\hline Zizyphus jujuba & Chinese date & Rhamnaceae & $\mathrm{N}^{1} \mathrm{P}^{3}$ & Fruit, Fodder, Oilseed \\
\hline Eriobotrya japonica & Loquat & Rosaceae & $\mathrm{N}^{1} \mathrm{P}^{1}$ & Fruit \\
\hline \multicolumn{5}{|l|}{ October } \\
\hline Adhatoda vasica & Basuti & Acanthaceae & $\mathrm{N}^{2} \mathrm{P}^{2}$ & - \\
\hline Agave americana & Century plant & Agavaceae & $\mathrm{N}^{3} \mathrm{P}^{3}$ & - \\
\hline Aster spp. & Star-wort & Asteraceae & $\mathrm{N}^{3} \mathrm{P}^{3}$ & Ornamental \\
\hline Chenopodium album & White goose foot & Chenopodiaceae & $\mathrm{N}^{3} \mathrm{P}^{3}$ & Fodder \\
\hline Ipomoea batatas & Sweet potato & Convolvulaceae & $\mathrm{N}^{2} \mathrm{P}^{3}$ & Vegetable \\
\hline Ipomoea pulchella & Railway creeper & Convolvulaceae & $\mathrm{N}^{3} \mathrm{P}^{3}$ & - \\
\hline Cuscuta reflexa & Amarbel & Convolvulaceae & $\mathrm{P}^{3}$ & Medicinal \\
\hline Leucaena leucocephala & Ipil-ipil & Fabaceae & $\mathrm{N}^{3} \mathrm{P}^{1}$ & Fuel, Fodder \\
\hline Hypericum spp. & Hypericum & Hypericaceae & $\mathrm{P}^{1}$ & $\begin{array}{c}\text { Twigs used as toothbrush, seed as condiment for } \\
\text { flavouring curries }\end{array}$ \\
\hline Mentha viridis & Mint & Lamiaceae & $\mathrm{N}^{3}$ & Aromatic herb \\
\hline Salvia spp. & Red sage & Lamiaceae & $\mathrm{N}^{2} \mathrm{P}^{2}$ & Medicinal \\
\hline Asphodelus tenuifolius & Piazi & Liliaceae & $\mathrm{N}^{3} \mathrm{P}^{2}$ & $\mathrm{C}^{\mathrm{N}}-$ \\
\hline Althaea rosea & Hollyhock & Malvaceae & $\mathrm{N}^{2} \mathrm{P}^{2}$ & Ornamental \\
\hline Malva sylvestris & Mallow & Malvaceae & $\mathrm{N}^{2} \mathrm{P}^{3}$ & Ornamental \\
\hline Malvaviscus arboreus & Malvaviscus & Malvaceae & $\mathrm{N}^{2} \mathrm{P}^{2}$ & Ornamental \\
\hline Musa sapientum & Banana & Musaceae & $\mathrm{N}^{2} \mathrm{P}^{3}$ & Fruit \\
\hline Callistemon citrinus & Bottle brush & Myrtaceae & $\mathrm{N}^{1} \mathrm{P}^{1}$ & Ornamental \\
\hline Rumex spp. & Rumex & Polygonaceae & $\mathrm{N}^{3} \mathrm{P}^{3}$ & - \\
\hline \multicolumn{5}{|l|}{ November } \\
\hline Adhatoda vasica & Basuti & Acanthaceae & $\mathrm{N}^{2} \mathrm{P}^{2}$ & - \\
\hline Agave americana & Century plant & Agavaceae & $\mathrm{N}^{3} \mathrm{P}^{3}$ & - \\
\hline Aster spp. & Star-wort & Asteraceae & $\mathrm{N}^{3} \mathrm{P}^{3}$ & - \\
\hline Cosmos sulphureus & Cosmos & Asteraceae & $\mathrm{N}^{3} \mathrm{P}^{2}$ & - \\
\hline Ipomoea batatas & Sweet potato & Convolvulaceae & $\mathrm{N}^{2} \mathrm{P}^{3}$ & Vegetable \\
\hline Ipomoea pulchella & Railway creeper & Convolvulaceae & $\mathrm{N}^{3} \mathrm{P}^{3}$ & - \\
\hline Bambusa bambos & Bamboo & Poaceae & $\mathrm{N}^{3} \mathrm{P}^{3}$ & Forest, Wood, Furniture, \\
\hline Camellia sinensis & Tea & Theaceae & $\mathrm{N}^{1} \mathrm{P}^{1}$ & Colouring material, Beverages \\
\hline \multicolumn{5}{|l|}{ December } \\
\hline Brassica campestris & Mustard & Brassicaceae & $\mathrm{N}^{1} \mathrm{P}^{1}$ & Oilseed \\
\hline Brassica napus var. glauca & Rape & Brassicaceae & $\mathrm{N}^{1} \mathrm{P}^{1}$ & Oilseed crop, Vegetable \\
\hline Brassica napus var. toria & Toria & Brassicaceae & $\mathrm{N}^{1} \mathrm{P}^{1}$ & Oilseed crop, Vegetable \\
\hline Eruca sativa & Rocket salad & Brassicaceae & $\mathrm{N}^{1} \mathrm{P}^{1}$ & Oilseed, Fodder \\
\hline Malvaviscus arboreus & Malvaviscus & Malvaceae & $\mathrm{N}^{2} \mathrm{P}^{2}$ & Ornamental \\
\hline
\end{tabular}

$\mathrm{N}^{1}=$ Major nectar source $\mathrm{P}^{1}=$ Major pollen source

$\mathrm{N}^{2}=$ Medium nectar source $\mathrm{P}^{2}=$ Medium pollen source

$\mathrm{N}^{3}=$ Minor nectar source $\mathrm{P}^{3}=$ Minor pollen source 


\section{Results and discussion}

The bee flora for Apis species recorded in different months of the year is given in the Table.

Most of the bee attracting plants provided nectar and pollen for the honey bees. But some plants were visited either for pollen or for nectar only. Early in spring (January-February) the bees worked mainly on Cruciferous crops. During late February to April, citrus species, shisham, curry leaf plant, pumpkin, litchi, mango, roses and bottle brush provided abundant nectar and pollen. Berseem came into bloom in late March and attracted sufficient bees for nectar and pollen upto June.

Late in June through August there was comparatively less bee forage. Bees collected nectar from cucurbits, sunflower, bhindi, cotton and pollen from maize and bajra, sown as early fodder grain crop. After the rainy season, i.e., during September and October, cotton, arhar, toria, maize and sunflower provided both nectar and pollen. Towards the onset of winter (November-December), sarson, toria, rocket salad were found to be the major bee attracting plants.

In shiwalik hills, there were two main nectar flow periods, viz., March-May and September-November. Brassica species was the major source of nectar during the first while cotton, bottle brush and basuti were quiet significant during the latter flows. There was no definite dearth period for the honey bees in the area. Although late June to August there was comparatively less bee forage, yet honey bees continued their activity on maize, cotton, marigold, chrysanthemum and sunflower to overcome this hot and humid spell. A short dearth period during September was terminated when sarson flowers become available in October. Thus there was no serious gap in the sequence flowering of bee flora in Shiwalik hills.

\section{Future Scope}

Assessment of bio resources including honey plant resources and their utilization pattern for hilly and plain area of NorthWest Himalayas. Floral maps should be prepared for whole of the Himalayan region. Detailed ecological and biochemical studies should be conducted on excellent nectar sources. Assessment of bio resources including honey plant resources and their utilization pattern for hilly and plain area of North-West Himalayas. For double-fold benefit, area based scientific beekeeping should be encouraged among the farmers. Need based research activities should be taken up.

\section{References}

[1] Crane, E. and Walker, P. 1984. Pollination Directory for World crops. IBRA. London, $\mathbf{1 8 3}$ pp.

[2] Deodikar, G.B. and Suryanarayana, M.C. 1972. Crop yield and bee pollination. Indian Bee J. 34: 53-63.

[3] Mattu, V.K. 1982. Morphometric and behavioural studies on Indian honeybee (Apis cerana F.). Ph. D. Thesis. Himachal Pradesh University, Shimla, India.
[4] McGregor, S.A. 1976. Insect pollination of cultivated crop plants. USDA/ARC Agric. Washington, DC handbook, 496 pp.

[5] Suryanarayana, M.C. 1978. Bee plants of India, Carvia callosa. Indian Bee J. 40: 7-10.

\section{Author Profile}

V. K. Mattu, professor at Department of Biosciences, Himachal Pradesh University, Shimla

Avinash Kaur, Ph.D. research scholar at Department of Biosciences, Himachal Pradesh University, Shimla

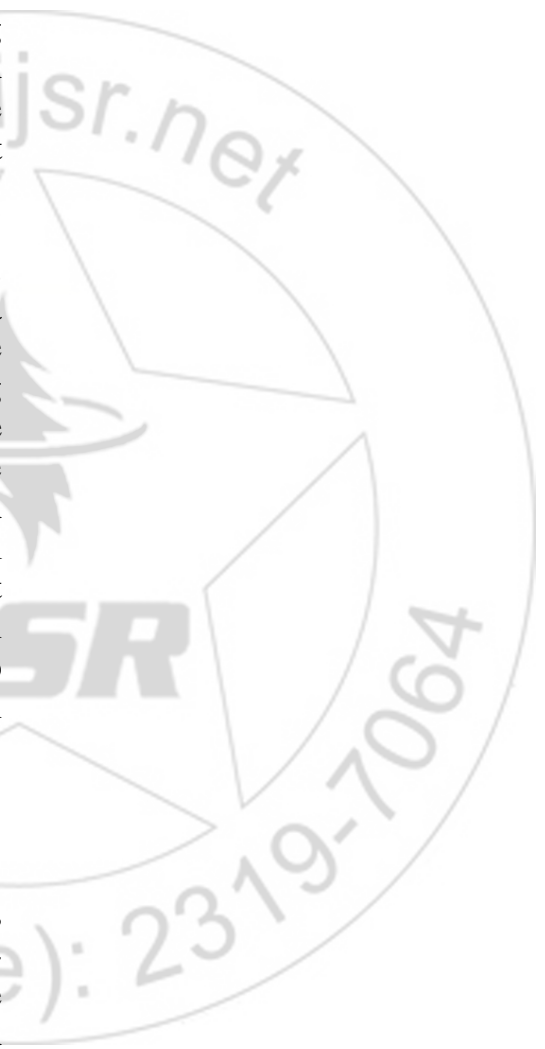

